\title{
Downregulation of NPM expression by Her-2 reduces resistance of gastric cancer to oxaliplatin
}

\author{
ZHENNI SUN ${ }^{1 *}$, LU YUE $^{1 *}$, ZAN SHEN $^{2}$, YONG LI $^{1}$, AIHUA SUI $^{3}$, \\ TIANJUN $\mathrm{LI}^{3}$, QIAN TANG ${ }^{3}$, RUYONG YAO ${ }^{3}$ and YONGNING SUN ${ }^{2}$
}

${ }^{1}$ Department of Oncology, Qingdao Municipal Hospital, School of Medicine, Qingdao University, Qingdao, Shandong 266003;

${ }^{2}$ Department of Oncology, The Sixth People's Hospital, Medical College of Shanghai Jiao Tong University, Shanghai 200233; ${ }^{3}$ Center for Medical Research, The Affiliated Hospital of Qingdao University, Qingdao, Shandong 266003, P.R. China

Received June 24, 2015; Accepted September 15, 2016

DOI: $10.3892 / 01.2017 .5722$

\begin{abstract}
Nucleophosmin (NPM) and human epidermal growth factor receptor-2 (Her-2) are abnormally expressed in various types of human malignant tumors, including gastric cancer, and have been closely associated with cancer chemoresistance. However, their interaction and roles in oxaliplatin resistance are not fully understood. Therefore, the present study aimed to elucidate the relationship between NPM and Her-2 in gastric cancer cell lines and clinical samples, and further investigated their role in the resistance of gastric cancer to oxaliplatin. Western blotting and reverse transcription-quantitative polymerase chain reaction confirmed that NPM and Her-2 expression were significantly upregulated in gastric cancer cells and clinical samples, and that their expression levels were strongly correlated. However, Her-2 expression was not affected by upregulation or downregulation of NPM expression in gastric cancer cells. Cell counting kit- 8 assays demonstrated that the cell sensitivity to oxaliplatin decreased simultaneously with an increase in NPM expression. Furthermore, inhibition of Her-2 expression using trastuzumab significantly increased the sensitivity of the cells to oxaliplatin, which occurred simultaneously with the downregulation of NPM. These results indicated that inhibition of NPM, as a Her-2 downstream signal, may be a novel strategy to overcome oxaliplatin-resistant gastric cancer, and
\end{abstract}

Correspondence to: Professor Ruyong Yao, Center for Medical Research, The Affiliated Hospital of Qingdao University, 16 Jiangsu Road, Qingdao, Shandong 266003, P.R. China

E-mail: yry0303@163.com; yongning_sun@163.com

Dr Yongning Sun, Department of Oncology, The Sixth People's Hospital, Medical College of Shanghai Jiao Tong University, 600 Yishan Road, Shanghai 200233, P.R. China

E-mail: yongning_sun@163.com

${ }^{*}$ Contributed equally

Key words: nucleophosmin, human epidermal growth factor receptor-2, drug resistance, gastric cancer that trastuzumab and oxaliplatin may exhibit a synergistic antitumor effect in Her-2-positive gastric cancer cells.

\section{Introduction}

Gastric cancer is the fifth most common malignancy and the third leading cause of cancer-associated mortality worldwide (1). According to GLOBOCAN 2012, 1 million new gastric cancer cases, with an $\sim 72 \%$ mortality rate, were diagnosed in 2012, of which 50\% occurred in Eastern Asia (predominantly in China) (2). The majority of patients present with an advanced stage of the disease at the time of diagnosis and, for them, palliative chemotherapy is the main choice of treatment (3). Oxaliplatin, which is a third-generation platinum analog, is widely used as a first-line chemotherapeutic agent for advanced gastric cancer (4). However, many patients eventually develop resistance after several courses of oxaliplatin-based chemotherapy, although the mechanisms remain poorly understood. Therefore, identification of suitable factors for predicting chemosensitivity or reducing resistance to oxaliplatin may be important for improving the therapeutic effect of oxaliplatin.

Nucleophosmin (NPM), which is also known as B23, numatrin or NO38, is a ubiquitously expressed nucleolar phosphoprotein that shuttles between the nucleus and cytoplasm (5). As a molecular chaperone, it is indispensable for various cellular processes, including ribosome biogenesis, centrosome duplication, DNA repair and stress responses (6). NPM is capable of binding to numerous partners in distinct cellular compartments and functions to maintain the dynamic balance between proliferation and apoptosis (7). Therefore, dysregulation of NPM expression and/or localization may contribute to tumorigenesis through various mechanisms. Previous studies have reported that NPM is highly expressed in proliferating cells and is involved in the tumorigenesis of hepatic (8), thyroid (9), gastric (10) and bladder (11) cancers. In addition, its upregulation in tumors has been correlated with a poor outcome (12). NPM is redistributed from the nucleus in response to cytotoxic drugs and genotoxic stress caused by inhibition of RNA polymerase I, exposure to DNA intercalating agents and UV damage $(13,14)$. Furthermore, the upregulation of NPM in breast cancer cell lines was shown 
to promote resistance to estrogen (15). Conversely, silencing of NPM expression sensitized hepatoma cells to doxorubicin, cisplatin, lapatinib, sorafenib and UV-B (16); thus suggesting that the inhibition of NPM may be considered a novel therapeutic strategy for cancer treatment.

Human epidermal growth factor receptor-2 (Her-2) is a member of the epidermal growth factor receptor family of receptor tyrosine kinases, which have vital roles in the development and progression of various solid tumors, including gastric cancer (17). Since the downregulation of Her-2 was shown to promote the apoptosis of tumor cells (18), Her-2 is regarded as a potential target in breast and gastric cancer therapy. It has been suggested that malignant tumors with high expression levels of Her-2 may be more aggressive and more resistant to chemotherapy (19). The Her-2-specific antibody, trastuzumab (also known as Herceptin), is currently approved for the treatment of Her-2-positive breast cancer and metastatic gastric cancer as a result of the Trastuzumab for Gastric Cancer (ToGA) trial $(20,21)$. As NPM and Her-2 have been shown to have overlapping roles in cell proliferation, apoptosis and gastric cancer development, and are considered therapeutic targets (22), it can be hypothesized that Her-2 and NPM expression may be correlated in the development of gastric cancer. The relationship and effect of NPM and Her-2 in chemoresistance require further elucidation.

The present study aimed to demonstrate the correlation between NPM and Her-2 expression in gastric cancer tissues and cell lines, as well as their role in the response of gastric cancer to oxaliplatin. In addition, the efficiency of using trastuzumab in combination with oxaliplatin for the treatment of gastric cancer was investigated, in order to provide a novel chemotherapeutic strategy for gastric cancer patients in clinical practice.

\section{Materials and methods}

Patients and clinical samples. Primary tumor tissues were obtained from 133 patients with gastric cancer who underwent surgical resection at the Department of General surgery, the Affiliated Hospital of Qingdao University (Qingdao, China) between January 2007 and November 2014. Patients enrolled in the study included 55 females and 78 males with an age range of 33-78 years (mean age, 61.4 years). No patients received chemotherapy or radiotherapy prior to surgery. In addition, 68 paracancer tissues that were located $>5 \mathrm{~cm}$ from the edge of tumor were obtained. All samples were fixed in formaldehyde for $24 \mathrm{~h}$, and then embedded in paraffin. All tumor tissue samples were confirmed to be adenocarcinoma histologically and the pathological diagnoses and stages were defined according to the International Union against Cancer tumor-node-metastasis (TNM) classification of malignant tumors (23). The study protocol was approved by the Ethics Committee of the Affiliated Hospital of Qingdao University. Informed consent was obtained from all patients.

Immunohistochemistry (IHC). IHC was performed to detect the expression of NPM and Her-2 in the gastric cancer and paired paracancer tissues. The paraffin-embedded tissues were cut into $5-\mu \mathrm{m}$ sections, deparaffinized and hydrated in graded ethanol. For antigen retrieval, the sections were incubated in citrate buffer solution and heated in a microwave oven (pH 6.0) for $20 \mathrm{~min}$. Endogenous peroxidase activity was inactivated by treating the sections with $0.3 \%$ hydrogen peroxide in methanol for $15 \mathrm{~min}$. After washing in PBS, the sections were incubated with the primary antibodies against NPM (dilution 1:100; catalog no., ab180607; Abcam, Cambridge, MA, USA) and Her-2 (dilution 1:200; catalog no., ab178176; Abcam) overnight at $4^{\circ} \mathrm{C}$. Antibody staining was detected using the avidin-biotin-peroxidase complex (PV9005; Zhongshan Jinqiao Biological Technology Co., Ltd., Beijing, China). Finally, sections were incubated in 3,3'-diaminobenzidine tetrahydrochloride for 3-10 min and counterstained with $0.1 \%$ hematoxylin. IHC staining was evaluated by two independent pathologists blinded to the clinicopathological characteristics according to the semiquantitative immunoreactivity score system, which involved measuring the intensity of staining (score 0-3) and the extent of staining (score 0,0\%; score 1, $0-10 \%$; score $2,10-50 \%$; score $3,50-100 \%$ ). The final weighted score (0-9) for each sample, which was obtained by multiplying the scores for the intensity and extent of staining, was divided into two groups: The positive group with scores of 4-9 and the negative group with scores of $0-3$.

Cell lines and cell culture. A total of four gastric cancer cell lines (SGC-7901, AGS, NCI-N87 and MKN-28) and two normal gastric epithelium cell lines (GES-1 and HFE-145), which show different expression levels of NPM and Her-2, were obtained from the Center for Medical Research of The Affiliated Hospital of Qingdao University (Qingdao, China). All cell lines were cultured in RPMI-1640 medium (Thermo Fisher Scientific, Inc., Waltham, MA, USA) supplemented with $10 \%$ fetal bovine serum (Hangzhou Sijiqing Biological Engineering Materials Co., Ltd., Hangzhou, China) and 1\% penicillin/streptomycin at $37^{\circ} \mathrm{C}$ in $5 \% \mathrm{CO}_{2}$.

Plasmid and transfection. To explore the relationship between NPM and Her-2, the expression of Her-2 or NPM in the cell lines was altered using the human Her-2 expression vector (pcDNA3.1-Her-2), Her-2-small interfering (si) RNA, the human NPM expression vector (pcDNA3.1-NPM) or NPM-siRNA. Briefly, cells were transfected in six-well plates $\left(5 \times 10^{5}\right.$ cells/well) with pcDNA3.1-control, control siRNA, pcDNA3.1-Her-2, Her-2-siRNA, pcDNA3.1-NPM or NPM-siRNA using Lipofectamine 2000 (Invitrogen; Thermo Fisher Scientific, Inc.), according to the manufacturer's protocol. Following transfection, the cells were incubated for 48-72 $\mathrm{h}$ in their normal media and the transfection efficiency was confirmed under microscope and by western blot analysis. Furthermore, following NPM transfection, the NPM overexpressing cells (AGSox) were incubated with oxaplatin (Sigma-Aldrich; EMD Millipore, Billerica, MA, USA) alone $(2.4 \mu \mathrm{g} / \mathrm{ml})$ or in combination with $5 \mu \mathrm{g} / \mathrm{ml}$ trastuzumab (a monoclonal antibody that interferes with the Her-2 receptor) for $24 \mathrm{~h}$ and subsequently were used for oxaliplatin resistance analysis.

RNA extraction and reverse transcription-quantitative polymerase chain reaction $(R T-q P C R)$. Total RNA was isolated from the four gastric cancer cells and the two normal gastric epithelium cells using TRIzol reagent (Invitrogen; 
Thermo Fisher Scientific, Inc.), according to the manufacturer's protocol, and then quantification and purity assessment (A260/A280 $\geq 1.8$ ) were performed using a spectrophotometer. RNA was reverse transcribed into cDNA using reverse transcriptase (Invitrogen; Thermo Fisher Scientific, Inc.), after which qPCR was performed on the LightCycler 480 Instrument II (Roche Diagnostics, Basel, Switzerland) using SYBR Premix Ex Taq reagents from the TaKaRa RNA PCR kit (Takara Bio, Inc., Otsu, Japan) according to the manufacturer's protocol. The primer sequences for PCR amplification were as follows: Her-2 (230 bp) sense, 5'-TCTGCTGGAGGAATTGGT GT-3' and antisense, 5'-GACGCTTGTTCCCACCTTGA-3'; NPM (310 bp) sense, 5'-UGUCUGUACAGCCAACGGUTT-3' and antisense, 5'-ACCGUUGGCUGUACAGACATT-3'; and $\beta$-actin (135 bp) sense, 5'-ACCACAGTCCTGCATGCCAC-3' and antisense, 5'-TCCACCACCCTGTTGCTGTA-3'. The PCR thermal cycling conditions were as follows: One cycle at $95^{\circ} \mathrm{C}$ for $10 \mathrm{sec}$, followed by 40 cycles at $95^{\circ} \mathrm{C}$ for $5 \mathrm{sec}$ and $60^{\circ} \mathrm{C}$ for $20 \mathrm{sec}$. Fold-changes in the mRNA expression levels of Her-2 and NPM were calculated following normalization to $\beta$-actin using the $2^{-\Delta \Delta \mathrm{Cq}}$ method (24). To ensure experimental accuracy, all reactions were performed in triplicate. An RT-mius control was performed using ultrapure water. Normal tissue served as a negative control.

Western blot analysis. Cells were collected and lysed using radioimmunoprecipitation assay lysis buffer containing $0.01 \%$ phenylmethylsulfonyl fluoride, $150 \mathrm{nmol} / 1$ Tris $(\mathrm{pH} 8)$, $0.1 \%$ SDS, $0.2 \%$ EDTA, $1 \%$ Triton $\mathrm{X}-100$ and $1 \%$ sodium deoxycholate, and supplemented with protease inhibitor and phosphatase inhibitor cocktails (Roche Diagnostics). The protein concentrations of the cellular extracts were determined using the bicinchoninic acid protein assay (Nanjing KeyGen Biotech, Co., Ltd., Nanjing, China), according to the manufacturer's protocol. Equal quantities (30-50 $\mu \mathrm{g})$ of proteins were loaded onto an 8-12\% SDS-polyacrylamide separating gel and transferred onto a polyvinylidene difluoride membrane (EMD Millipore, Billerica, MA, USA). The membrane was blocked with $1 \%$ bovine serum albumin (Shanghai Qcbio Science \& Technologies Co., Ltd., Shanghai, China) in TBST and then incubated at $4^{\circ} \mathrm{C}$ overnight with anti-NPM (dilution 1:1,000; catalog no., 20314263-2; Bioworld Technology, Inc., St. Louis Park, MN, USA), anti-Her-2 (dilution 1:2,000; catalog no., ab178176; Abcam) and anti-GAPDH (dilution 1:2,000; catalog no., CW0100M; Beijing ComWin Biotech Co., Ltd. Beijing, China) rabbit polyclonal antibodies, followed by appropriate horseradish peroxidase-conjugated secondary antibodies (dilution, 1:500; catalog. no., SH026; Applied Biological Materials, Inc., Richmond, BC, Canada). Antibody staining was visualized by enhanced chemiluminescence (EMD Millipore) and the images were analyzed using Quantity One 4.31 software (Bio-Rad Laboratories, Inc., Hercules, CA, USA).

Cell counting kit (CCK)-8 assay. The sensitivity of the gastric cancer cells with different expression levels of NPM to oxaliplatin were detected using the CCK-8 assay kit (Dojindo Molecular Technologies, Inc., Kumamoto, Japan). Briefly, cells were seeded into 96-well culture plates (10,000 cells/well) in $100 \mu \mathrm{l}$ fresh medium containing various concentrations of oxaliplatin $(0.3,0.6,1.2,2.4,4.8,9.6$ or $19.2 \mu \mathrm{g} / \mathrm{ml})$. After a
Table I. Differential expression of Her-2 and NPM in gastric cancer tissues and non-cancerous tissues.

\begin{tabular}{|c|c|c|c|c|}
\hline \multirow[b]{2}{*}{ Tissue } & \multicolumn{2}{|c|}{ NPM } & \multicolumn{2}{|c|}{ Her-2 } \\
\hline & + & - & + & - \\
\hline Gastric cancer & $89(66.9)$ & $44(33.1)$ & $21(15.8)$ & $112(84.2)$ \\
\hline Paracancer & $12(17.7)$ & $56(82.4)$ & $4(5.9)$ & $64(94.1)$ \\
\hline$\chi^{2}$ & \multicolumn{2}{|c|}{43.69} & \multicolumn{2}{|c|}{4.05} \\
\hline P-value & \multicolumn{2}{|c|}{$<0.0001$} & \multicolumn{2}{|c|}{$<0.05$} \\
\hline
\end{tabular}

Data are presented as n (\%). NPM, nucleophosmin; Her-2, human epidermal growth factor receptor- 2 .

Table II. Correlation between the expression of NPM and Her-2 in gastric cancer tissues only.

\begin{tabular}{lccc}
\hline Variable & Her-2(+) & Her-2(-) & P-value \\
\hline $\operatorname{NPM}(+)$ & $24(96.0)$ & $75(43.1)$ & $<0.0001$ \\
$\operatorname{NPM}(-)$ & $1(4.0)$ & $99(56.9)$ & \\
\hline
\end{tabular}

Data are presented as n (\%). NPM, nucleophosmin; Her-2, human epidermal growth factor receptor- 2 .

24-h incubation, $10 \mu \mathrm{l} \mathrm{CCK-8} \mathrm{reagent} \mathrm{was} \mathrm{added} \mathrm{to} \mathrm{each} \mathrm{well}$ at $37^{\circ} \mathrm{C}$ for $4 \mathrm{~h}$. The microplate reader (Bio-Rad Model 550; Bio-Rad Laboratories, Inc.) was used to determine the optical density (OD) of each well at $450 \mathrm{~nm}$. CCK-8 assays were repeated three times on different days to verify the accuracy. The cell inhibition rate was calculated using the following formula: Cell inhibition rate $(\%)=[1-(\mathrm{OD}$ experiment $-\mathrm{OD}$ blank) / (OD control - OD blank)] x 100. The half maximal inhibitory concentration $\left(\mathrm{IC}_{50}\right)$ of oxaliplatin was obtained by interpretation of the graph.

Statistical analysis. Quantitative data are expressed as the mean \pm standard deviation. Statistical comparisons were performed by one-way analysis of variance and $\chi^{2}$ tests as appropriate. The SAS 9.0 software (SAS Inc., Chicago, IL, USA) was used for statistical analysis. $\mathrm{P}<0.05$ was considered to indicate a statistically significant difference.

\section{Results}

Expression of Her-2 and NPM in gastric cancer tissues. Expression of NPM and Her-2 in gastric cancer tissues was measured by semiquantitative IHC analyses (Fig. 1). NPM was localized predominantly in the nucleoli, nuclei and cytoplasm and showed different intensities and extents of staining; the positive expression rates in the cancer tissues and paracancer tissues were 66.9 and $17.7 \%$, respectively. Conversely, Her-2 was expressed mainly in the membrane or cytoplasm of tumor epithelial cells, and the positive expression rates in the cancer tissues and paracancer tissues were 15.8 and $5.9 \%$, respectively (Table I). These results showed that the expression levels 

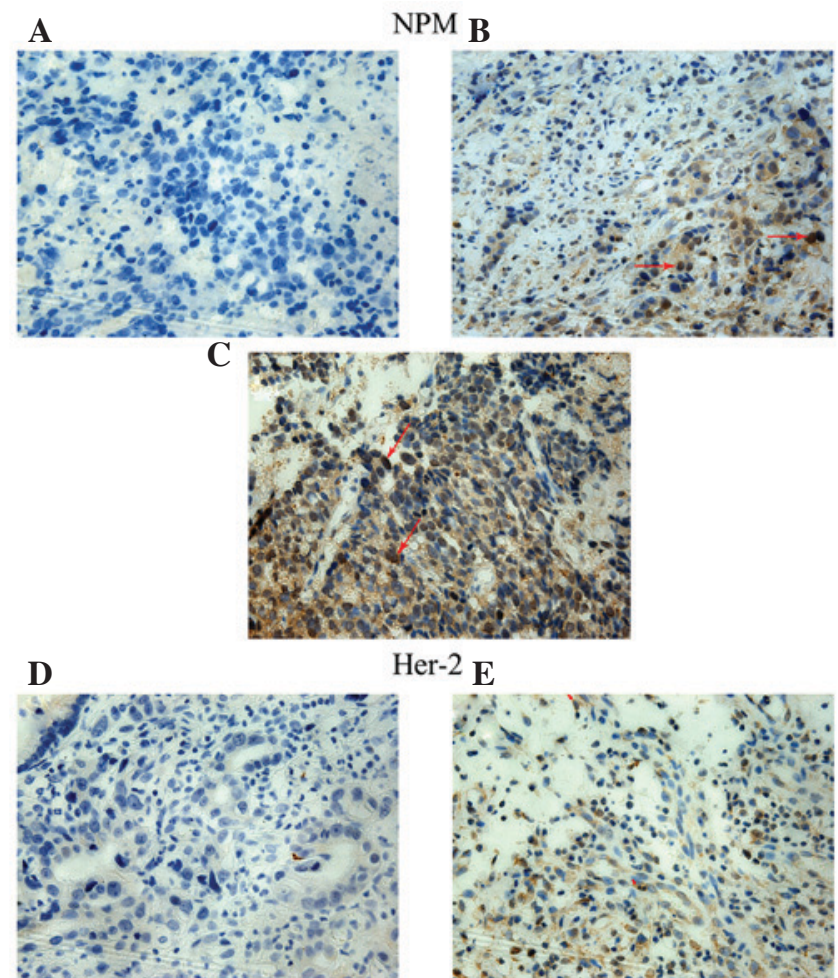

Her-2 $\mathbf{E}$

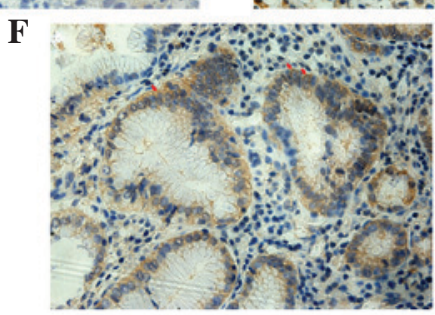

Figure 1. Expression of Her-2 and NPM in gastric cancer tissues. (A-C) NPM localized predominantly to the nucleoli, nuclei and cytoplasm of gastric cancer cells to varying intensities and degrees (indicated by long red arrows). (A) Negative expression of NPM. (B) Low intensity and percentage of NPM positive expression. (C) High intensity and percentage of NPM positive expression. (D-F) Her-2 was expressed predominantly in the membrane or cytoplasm of tumor epithelial cells (indicated by short red arrows) (D) Negative expression of Her-2. (E) Low intensity and percentage of Her-2 positive expression. (F) High intensity and percentage of Her-2 positive expression. Magnification, x200. NPM, nucleophosmin; Her-2, human epidermal growth factor receptor-2.

of NPM and Her-2 were significantly higher in gastric cancer tissues than in the paracancer tissues $(\mathrm{P}<0.01$ and $\mathrm{P}<0.05$, respectively). In addition, $24(96 \%)$ gastric carcinoma tissues exhibited positive Her-2 and NPM expression, and it was demonstrated there was a significant and positive correlation between NPM and Her-2 expression ( $\mathrm{P}<0.0001$; Table II).

NPM and Her-2 are overexpressed in gastric cancer cell lines. In our previous study, it was observed that the Her- 2 expression level altered in accordance with the NPM expression level following NPM gene transfection (22), which suggested that there is a relationship between NPM and Her-2 in gastric cancer. In the present study, the expression levels of NPM and Her-2 were detected in four gastric cancer cell lines and two normal gastric epithelium cell lines using RT-qPCR and western blot analysis. The expression of the NPM protein, as detected using an NPM-specific antibody, correlated with

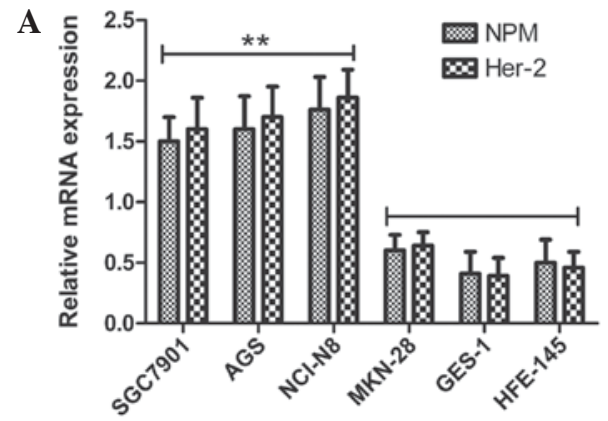

B

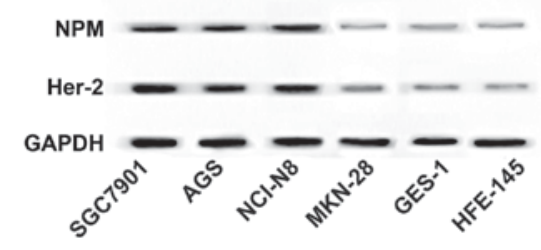

C

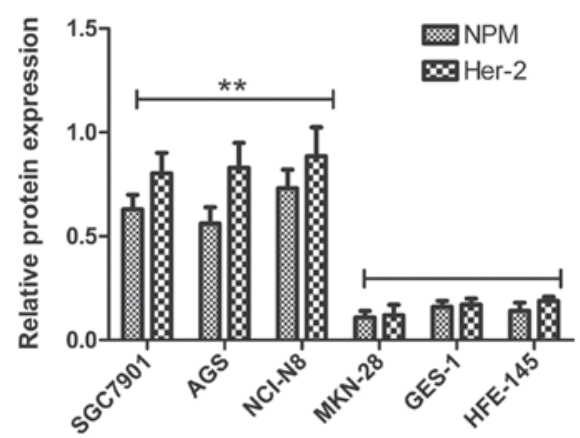

Figure 2. Expression of NPM and Her-2 in different gastric cell lines. (A) NPM and Her-2 mRNA expression levels in different gastric cancer or epithelium cell lines were determined by reverse transcription-quantitative polymerase chain reaction. (B) Protein expression of NPM and Her-2 in gastric cells was determined by western blotting. (C) Relative protein expression levels of NPM and Her-2 in different gastric cells were quantified by densitometry. ${ }^{* *} \mathrm{P}<0.01$. NPM, nucleophosmin; Her-2, human epidermal growth factor receptor-2.

that of NPM mRNA in gastric cancer cell lines (Fig. 2). As compared with the normal gastric epithelium cell lines, NPM was significantly overexpressed in three of the four gastric cancer cell lines (Fig. 2A), suggesting that NPM may be considered a target for inhibition of gastric cancer cell activation. Furthermore, high mRNA and protein expression levels of Her-2 were detected in SGC7901, AGS and NCI-N87 cells, whereas both normal gastric epithelium cell lines exhibited low expression levels of Her-2 (Fig. 2B and C). The differences in the mRNA and protein expression levels of NPM and Her-2 between the malignant and benign gastric cells were significant, with the exception of the MKN-28 cell line.

Her-2 regulates NPM expression in gastric cancer cell lines. When further analyzing the relationship between NPM and HER-2 expression, it was demonstrated that the three gastric cancer cell lines that exhibited NPM overexpression were all positive for Her-2 expression, while the Her-2-negative gastric cancer cell line, MKN-28, also expressed low levels of NPM (Fig. 2). Altogether, all four gastric cancer cell lines exhibited a positive correlation between the expression levels of NPM and Her-2. In order to explore the interaction relationship 
A

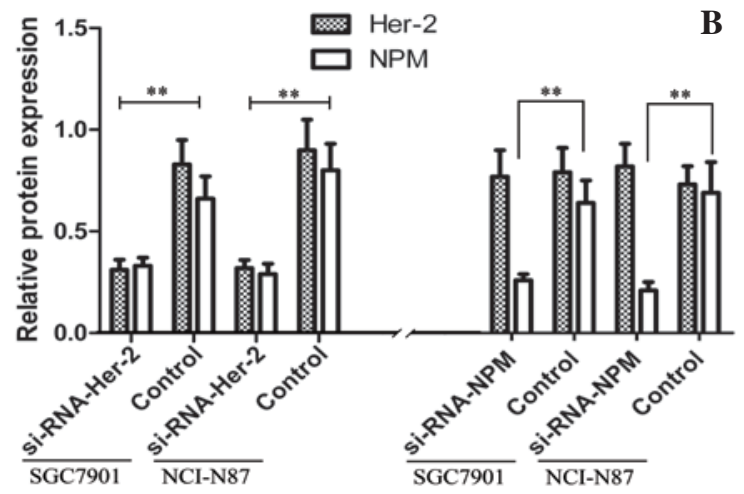

B

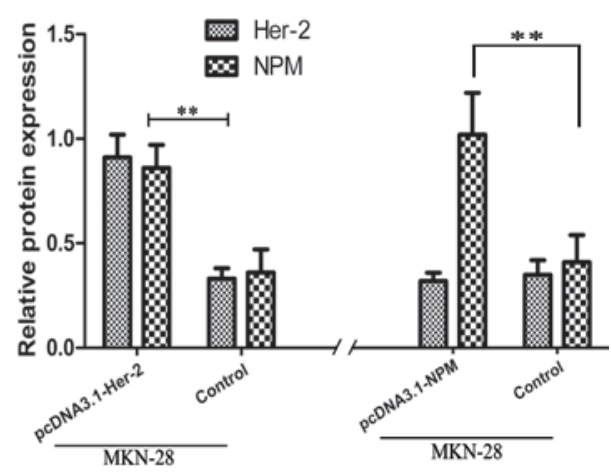

Figure 3. Her-2 regulates NPM expression in gastric cancer cell lines. (A) Downregulating Her-2 expression in gastric cancer cells using Her-2-specific siRNA altered NPM expression concordantly, while downregulating NPM expression using NPM-specific siRNA did not significantly alter Her-2 expression. (B) Upregulating Her-2 expression in MKN-28 gastric cancer cells using a Her-2-expressing plasmid altered NPM expression, while upregulating NPM expression in the same cells did not lead to a prominent change in Her-2 expression. ${ }^{* *} \mathrm{P}<0.01$. NPM, nucleophosmin; Her-2, human epidermal growth factor receptor-2; siRNA, small interfering RNA.
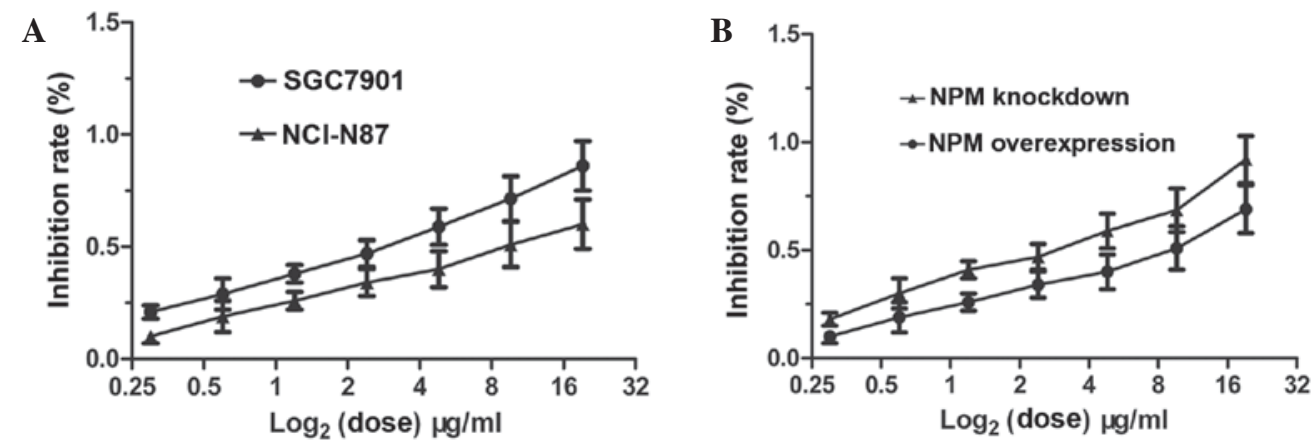

Figure 4. NPM overexpression correlates to resistance of gastric cancer cells to oxaliplatin, as determined using cell counting kit-8 assays. (A) NCI-N87 cells, which show higher expression of NPM, had a higher survival rate than SGC7901 cells, which exhibit lower NPM expression. (B) NPM overexpressing AGS cells had a significantly higher half maximal inhibitory value compared with the NPM-knockdown cells. NPM, nucleophosmin; Her-2, human epidermal growth factor receptor-2.

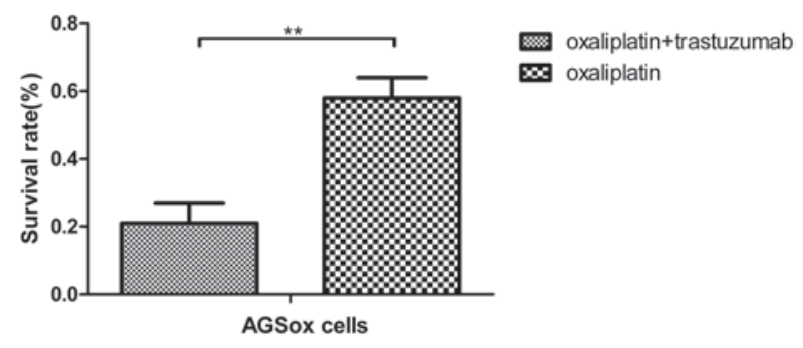

Figure 5. Trastuzumab sensitized gastric cancer cells to oxaliplatin in vitro. AGSox cells treated with oxaliplatin combined with trastuzumab exhibited a significantly lower survival rate than the cells treated with oxaliplatin alone. ${ }^{* *} \mathrm{P}<0.01$. AGSox cells, NPM-overexpressing AGS gastric cancer cells.

between Her-2 and NPM, transfection technology was used to alter their expression. First, SGC7901 and NCI-N87 cells were transfected with Her-2-siRNA or non-specific siRNA. Western blot analysis confirmed that Her-2 expression was successfully altered by the transfection, and that NPM expression changed in accordance with Her-2 downregulation (Fig. 3A). Conversely, no significant difference in the expression level of Her-2 was observed when NPM expression was silenced using NPM-siRNA (Fig. 3A). Furthermore, elevated NPM expression was observed following transfection of MKN-28 cells with pcDNA3.1-Her-2, whereas overexpression of NPM caused by transfection of the MKN-28 cells with pcDNA3.1-NPM did not lead to a prominent change in Her-2 expression (Fig. 3B). These results suggest that NPM expression in gastric cancer cells is regulated by Her- 2 .

NPM overexpression correlates with resistance to oxaliplatin. Considering the opposite effects of NPM and oxaliplatin on DNA, we hypothesized that NPM may affect the sensitivity of gastric cancer cells to oxaliplatin. Three gastric cancer cell lines (SGC7901, AGS and NCI-N87), which show different expression levels of NPM, were treated with serial concentrations of oxaliplatin for $24 \mathrm{~h}$, and the viability of these cells was assessed using CCK- 8 assays. The results revealed that the NCI-N87 cells had the highest survival rate and highest expression levels of NPM, while the SGC7901 cells exhibited the lowest survival rate and the lowest NPM expression (Fig. 4A), indicating that the expression of NPM was positively correlated with oxaliplatin resistance. To further validate the results, AGS cells were transfected with pcDNA3.1-NPM or NPM-siRNA, and western blot analysis revealed that the transfection efficiently altered NPM expression. As shown in Fig. 4B, the cell viability was markedly lower in NPM-knockdown cells, whereas the cells overexpressing NPM exhibited a higher viability. Furthermore, the cells overexpressing NPM 
had a significantly higher $\mathrm{IC}_{50}$ value, as compared with the NPM-knockdown cells (7.19 $\mu \mathrm{g} / \mathrm{ml}$ vs. $2.26 \mu \mathrm{g} / \mathrm{ml}$; Fig. 4B). These results suggest that NPM expression was associated with the therapeutic efficacy of oxaliplatin and that NPM may be a potential predictive factor of oxaliplatin resistance in gastric cancer.

Trastuzumab sensitizes gastric cancer cells to oxaliplatin in vitro. Considering the relationship between Her-2 and NPM, NPM-overexpressing cells (AGSox) were used to reveal the role of trastuzumab in the reversal of oxaliplatin resistance. The AGSox cells were incubated with oxaliplatin alone $(2.4 \mu \mathrm{g} / \mathrm{ml})$ or in combination with $5 \mu \mathrm{g} / \mathrm{ml}$ trastuzumab and then assessed for viability. The CCK- 8 assay results demonstrated that the AGSox cells treated with oxaliplatin combined with trastuzumab exhibited a significantly lower survival rate than the cells that experienced monotherapy ( 21 vs. 58\%, respectively; Fig. 5). These results suggest that there is a synergistic effect between the two drugs, and that the resistance of gastric cancer cells to oxaliplatin may be reversed through the inhibition of NPM via Her-2.

\section{Discussion}

The results of the present study provided evidence for a functional role of NPM in oxaliplatin resistance in human gastric cancer cells and clarified the relationship between NPM and Her-2 in gastric cancer, which has seldom been described. Notably, NPM and Her-2 were significantly upregulated in gastric cancer cell lines and clinical samples, as compared with two normal gastric epithelium cell lines and paracancerous tissues, respectively. Furthermore, a positive relationship between the expression levels of NPM and Her-2 was observed; when Her-2 expression was up- or down-regulated, the NPM expression was altered accordingly. Conversely, alterations in the expression levels of NPM did not significantly affect Her-2 expression, indicating that the expression of NPM was at least partially regulated by Her-2. In addition, NPM overexpression was shown to promote oxaliplatin resistance in gastric cancer cells and the Her-2-specific inhibitor trastuzumab was able to significantly reverse the oxaliplatin resistance.

Her-2 has an important role in promoting cell proliferation and suppressing apoptosis, and may facilitate uncontrolled cell growth and tumorigenesis by transmitting extracellular signals to initiate intracellular signaling pathways (25). Importantly, Her-2 overexpression has been described in a variety of tumors, including breast cancer, gastric cancer, non-small cell lung cancer and ovarian cancer (26). Overexpression of Her-2 at the protein and/or mRNA level is detected in a variable percentage of cases, depending on the tumor (27-29). Furthermore, unlike breast cancer, previous studies have reported inconsistent findings regarding the prognostic role of Her-2 in gastric cancer (30-32). A previous study demonstrated that patients with intestinal-type and well-differentiated gastric cancer have a higher rate of Her-2 positivity than patients with diffuse-type and poorly-differentiated cancer (29). It has been reported that the Her-2-positive status was associated with a significantly worse prognosis and to relevant clinicopathological characteristics, including already established prognostic factors such as serosal invasion and lymph node metastasis (33).
Ding et al (34) reported that NPM was an independent prognostic indicator for tumor recurrence in patients with gastric cancer, and was associated with advanced tumor stages and a poor prognosis. Our previous study demonstrated that NPM and Her-2 expression were closely related to a later TNM stage and the recurrence or metastasis of gastric cancer (22). However, the relationship between NPM and Her-2 has yet to be investigated. The results of the present study suggested that NPM expression was closely associated with the Her-2 expression status, and that NPM is a downstream cellular target of Her-2-dependent signaling. Furthermore, the results indicated that Her-2 and NPM may be important diagnostic markers for gastric cancer. However, the precise regulatory mechanisms underlying the interaction between Her-2 and NPM remain unclear.

Recurrence and subsequent acquired chemoresistance are responsible for therapeutic failure and poor improvement in the 5-year survival rate of gastric cancer patients (22). Oxaliplatin has been reported to induce apoptosis via various mechanisms, including downregulating the expression of anti-apoptotic proteins, influencing mitogen-activated protein kinase activities, inhibiting DNA-repair mechanisms and inducing mitotic catastrophe (35-38). However, chemoresistance has been reported for oxaliplatin (39), and is thought to occur as a result of Her-2 overexpression (40). Elucidating the mechanisms by which Her-2 mediates resistance to oxaliplatin may be crucial for improving therapeutic strategies. NPM is required for DNA-dependent activities, including transcription, replication and repair (41). On the basis of the opposite roles of NPM and oxaliplatin in the progression of DNA repair, the authors of the present study hypothesized that NPM may be involved in the resistance of gastric cancer to oxaliplatin and further examined this possibility. The present study found that NPM overexpression was closely related to oxaliplatin resistance in gastric cancer cells, whereas silencing of NPM with siRNA was able to significantly increase the chemosensitivity of gastric cancer cells, indicating that NPM may be a potential therapeutic target for oxaliplatin-resistant gastric cancer.

The present study also demonstrated that resistance to oxaliplatin induced by NPM overexpression was partially reversed by trastuzumab, which is an anti-Her-2 monoclonal antibody, indicating a synergistic antitumor effect for trastuzumab and oxaliplatin in Her-2-positive gastric cancer cells. Overexpression or amplification of Her-2 is detected in $7-34 \%$ of gastric cancer patients and, in a previous study, the addition of trastuzumab to chemotherapy improved survival in patients with advanced gastric cancer compared with chemotherapy alone (21). ToGA clinical trials in patients with gastric adenocarcinoma and gastroesophageal junction adenocarcinoma have shown a significant survival benefit for patients treated with a combination of trastuzumab and standard chemotherapy $(42,43)$. The results of a multicenter phase II clinical trial suggested that the combination of trastuzumab and XELOX (oxaliplatin and capecitabine) was highly efficacious, demonstrating an objective response rate of $68 \%$, a median progression-free survival (PFS) of 9.8 months and a median overall survival of 21.0 months; the PFS was enhanced by 2 months compared with previous phase II trials of XELOX alone $(44,45)$. Furthermore, trastuzumab showed synergistic cytotoxic effects with oxaliplatin on 
Her-2-positive gastric and breast cancer cells (46). However, adverse effects, including grade 3-4 peripheral neuropathy, neutropenia and anemia, were noted following combined treatment with trastuzumab and oxaliplatin (47). Therefore, further investigations are required to identify a more efficient and safe therapy.

In conclusion, the present study demonstrated that NPM may be a potential target for oxaliplatin-resistant gastric cancer. In addition, trastuzumab and oxaliplatin exhibited a synergistic antitumor effect in Her-2-postive gastric cancer cells.

\section{Acknowledgements}

This study was supported by the Shandong Natural Science Foundation (grant nos., ZR2015HM085 and ZR2015PH013).

\section{References}

1. Kalnina Z, Meistere I, Kikuste I, Tolmanis I, Zayakin P and Linē À: Emerging blood-based biomarkers for detection of gastric cancer. World J Gastroenterol 21: 11636-11653, 2015.

2. International Agency for Research on Cancer; World Health Organization: GLOBOCAN 2012: Estimated Cancer Incidence, Mortality and Prevalence Worldwide in 2012. http://globocan. iarc.fr/Default.aspx. Accessed February 1, 2015.

3. Zagouri F, Papadimitriou CA, Dimopoulos MA and Pectasides D: Molecularly targeted therapies in unresectable-metastatic gastric cancer: A systematic review. Cancer Treat Rev 37: 599-610, 2011

4. Montagnani F, Turrisi G, Marinozzi C, Aliberti C and Fiorentini G: Effectiveness and safety of oxaliplatin compared to cisplatin for advanced, unresectable gastric cancer: A systematic review and meta-analysis. Gastric Cancer 14: 50-55, 2011.

5. Colombo E, Alcalay M and Pelicci PG: Nucleophosmin and its complex network: A possible therapeutic target in hematological diseases. Oncogene 30: 2595-2609, 2011.

6. Grisendi S, Mecucci C, Falini B and Pandolfi PP: Nucleophosmin and cancer. Nat Rev Cancer 6: 493-505, 2006.

7. Box JK, Paquet N, Adams MN, Boucher D, Bolderson E, O'Byrne KJ and Richard DJ: Nucleophosmin: From structure and function to disease development. BMC Mol Biol 17: 19, 2016.

8. Yun JP, Miao J, Chen GG, Tian QH, Zhang CQ, Xiang J, Fu J and Lai PB: Increased expression of nucleophosmin/B23 in hepatocellular carcinoma and correlation with clinicopathological parameters. Br J Cancer 96: 477-484, 2007.

9. Pianta A, Puppin C, Passon N, Franzoni A, Romanello M, Tell G, Di Loreto C, Bulotta S, Russo D and Damante G: Nucleophosmin delocalization in thyroid tumour cells. Endocr Pathol 22: 18-23, 2011.

10. Tanaka M, Sasaki H, Kino I, Sugimura T and Terada M: Genes preferentially expressed in embryo stomach are predominantly expressed in gastric cancer. Cancer Res 52: 3372-3377, 1992.

11. Chen SH, Wang YW, Hsu JL, Chang HY, Wang CY, Shen PT, Chiang CW, Chuang JJ, Tsai HW, Gu PW, et al: Nucleophosmin in the pathogenesis of arsenic-related bladder carcinogenesis revealed by quantitative proteomics. Toxicol Appl Pharmacol 242: $126-135,2010$

12. Dang H, Chen Y, Kamel-Reid S, Brandwein J and Chang H: CD34 expression predicts an adverse outcome in patients with NPM1-positive acute myeloid leukemia. Hum Pathol 44: 2038-2046, 2013.

13. Wu MH, Chang JH and Yung BY: Resistance to UV-induced cell-killing in nucleophosmin/B23 over-expressed NIH 3T3 fibroblasts: Enhancement of DNA repair and up-regulation of PCNA in association with nucleophosmin/B23 over-expression. Carcinogenesis 23: 93-100, 2002.

14. Kurki S, Peltonen K, Latonen L, Kiviharju TM, Ojala PM, Meek D and Laiho M: Nucleolar protein NPM interacts with HDM2 and protects tumor suppressor protein p53 from HDM2-mediated degradation. Cancer Cell 5: 465-475, 2004.

15. Clarke R, Liu MC, Bouker KB, Gu Z, Lee RY, Zhu Y, Skaar TC, Gomez B, O'Brien K, Wang Y, et al: Antiestrogen resistance in breast cancer and the role of estrogen receptor signaling. Oncogene 22: 7316-7339, 2003.
16. Lo SJ, Fan LC, Tsai YF, Lin KY, Huang HL, Wang TH, Liu H, Chen TC, Huang SF, Chang CJ, et al: A novel interaction of nucleophosmin with BCL2-associated X protein regulating death evasion and drug sensitivity in human hepatoma cells. Hepatology 57: 1893-1905, 2013.

17. Okada K, Fujiwara Y, Takahashi T, Nakamura Y, Takiguchi S, Nakajima K, Miyata H, Yamasaki M, Kurokawa Y, Mori M and Doki Y: Overexpression of forkhead box M1 transcription factor (FOXM1) is a potential prognostic marker and enhances chemoresistance for docetaxel in gastric cancer. Ann Surg Oncol 20: 1035-1043, 2013

18. Tian C, Ding P, Yuan Z, Li H, Zhao Y, Sun L, Guo Q, Wang Z, Sun L, Zhang L and Jiang Z: A novel dual EGFR/HER2 inhibitor KU004 induces cell cycle arrest and apoptosis in HER2-overexpressing cancer cells. Apoptosis 20: 1599-1612, 2015.

19. Dos Santos ML, Gimenes KP, Silva WA Jr, and Nagai MA: Transcriptome changes induced by docetaxel in human mammary cell lines expressing different levels of ERBB2. Int J Mol Med 23: 733-743, 2009.

20. Tripathy D, Slamon DJ, Cobleigh M, Arnold A, Saleh M, Mortimer JE, Murphy M and Stewart SJ: Safety of treatment of metastatic breast cancer with trastuzumab beyond disease progression. J Clin Oncol 22: 1063-1070, 2004.

21. Bang YJ, Van Cutsem E, Feyereislova A, Chung HC, Shen L, Sawaki A, Lordick F, Ohtsu A, Omuro Y, Satoh T, et al: Trastuzumab in combination with chemotherapy versus chemotherapy alone for treatment of HER2-positive advanced gastric or gastro-oesophageal junction cancer (ToGA): A phase 3 , open-label, randomised controlled trial. Lancet 376: 687-697, 2010.

22. Zhou F, Qiu W, Sun L, Xiang J, Sun X, Sui A, Ding A and Yue L: Clinical significance of nucleophosmin/B23 and human epidermal growth factor receptor $2 /$ neu expressions in gastric cancers. APMIS 121: 582-591, 2013.

23. Sobin LH, Gospodarowicz MK and Wittekind Ch (eds): TNM Classification of Malignant Tumors. 7th edition. WileyBlackwell, Oxford, 2009.

24. Livak KJ and Schmittgen TD: Analysis of relative gene expression data using real-time quantitative PCR and the 2(-Delta Delta C(T)) Method. Methods 25: 402-408, 2001.

25. Yarden Y and Sliwkowski MX: Untangling the ErbB signalling network. Nat Rev Mol Cell Biol 2: 127-137, 2001.

26. Scholl S, Beuzeboc P and Pouillart P: Targeting HER2 in other tumor types. Ann Oncol 12 (Suppl 1): S81-S87, 2011.

27. Slamon DJ, Godolphin W, Jones LA, Holt JA, Wong SG, Keith DE, Levin WJ, Stuart SG, Udove J and Ullrich A: Studies of the HER-2/neu proto-oncogene in human breast and ovarian cancer. Science 244: 707-712, 1989.

28. Grob TJ, Kannengiesser I, Tsourlakis MC, Atanackovic D, Koenig AM, Vashist YK, Klose H, Marx AH, Koops S, Simon R, et al: Heterogeneity of ERBB2 amplification in adenocarcinoma, squamous cell carcinoma and large cell undifferentiated carcinoma of the lung. Mod Pathol 25: 1566-1573, 2012.

29. Oh HS, Eom DW, Kang GH, Ahn YC, Lee SJ, Kim JH, Jang HJ, Kim EJ, Oh KH and Ahn HJ: Prognostic implications of EGFR and HER-2 alteration assessed by immunohistochemistry and silver in situ hybridization in gastric cancer patients following curative resection. Gastric Cancer 17: 402-411, 2014

30. Mihmanli M, Ilhan E, Idiz UO, Alemdar A and Demir U: Recent developments and innovations in gastric cancer. World J Gastronenterol 22: 4307-4320, 2016

31. Uprak TK, Attaallah W, Celikel CA, Ayrancı G and Yeğen C: HER-2 incidence in gastric cancer, its association with prognosis and clinicopathological parameters. Ulus Cerrahi Derg 31: 207-213, 2015.

32. Boku N: HER2-positive gastric cancer. Gastric Cancer 17: 1-12, 2014.

33. Jørgensen JT and Hersom M: HER2 as a prognostic marker in gastric cancer-A systematic analysis of data from the literature. J Cancer 3: 137-144, 2012.

34. Ding A, Zhao W, Shi X, Yao R, Zhou F, Yue L, Liu S and Qiu W: Impact of NPM, TFF3 and TACC1 on the prognosis of patients with primary gastric cancer. PloS One 8: e82136, 2013.

35. Fodale V, Pierobon M, Liotta L and Petricoin E: Mechanism of cell adaptation: When and how do cancer cells develop chemoresistance? Cancer J 17: 89-95, 2011.

36. Liu HF, Hu HC and Chao JI: Oxaliplatin down-regulates survivin by p38 MAP kinase and proteasome in human colon cancer cells. Chem Biol Interact 188: 535-545, 2010. 
37. Allen JE and El-Deiry WS: Oxaliplatin uses JNK to restore TRAIL sensitivity in cancer cells through Bcl-xL inactivation. Gastroenterology 141: 430-434, 2011.

38. Zecevic A, Sampath D, Ewald B, Chen R, Wierda W and Plunkett W: Killing of chronic lymphocytic leukemia by the combination of fludarabine and oxaliplatin is dependent on the activity of XPF endonuclease. Clin Cancer Res 17: 4731-4741, 2011.

39. Ngan CY, Yamamoto H, Takagi A, Fujie Y, Takemasa I, Ikeda M, Takahashi-Yanaga F, Sasaguri T, Sekimoto M, Matsuura N and Monden M: Oxaliplatin induces mitotic catastrophe and apoptosis in esophageal cancer cells. Cancer Sci 99: 129-139, 2008.

40. Sclafani F, Roy A, Cunningham D, Wotherspoon A, Peckitt C, Gonzalez de Castro D, Tabernero J, Glimelius B, Cervantes A, Eltahir Z, et al: HER2 in high-risk rectal cancer patients treated in EXPERT-C, a randomized phase II trial of neoadjuvant capecitabine and oxaliplatin (CAPOX) and chemoradiotherapy (CRT) with or without cetuximab. Ann Oncol 24: 3123-3128, 2013

41. Wu MH and Yung BY: UV stimulation of nucleophosmin/B23 expression is an immediate-early gene response induced by damaged DNA. J Biol Chem 277: 48234-48240, 2002.

42. Bang YJ, Van Cutsem E, Feyereislova A, Chung HC, Shen L, Sawaki A, Lordick F, Ohtsu A, Omuro Y, Satoh T, et al; ToGA Trial Investigators: Trastuzumab in combination with chemotherapy versus chemotherapy alone for treatment of HER2-positive advanced gastric or gastro-oesophageal junction cancer (ToGA): A phase 3, open-label, randomized controlled trial. Lancet 376: 687-697, 2010.
43. Lordick F, Kang YK, Chung HC, Salman P, Oh SC, Bodoky G, Kurteva G, Volovat C, Moiseyenko VM, Gorbunova V, et al; Arbeitsgemeinschaft Internistische Onkologie and EXPAND Investigators: Capecitabine and cisplatin with or without cetuximab for patients with previously untreated advanced gastric cancer (EXPAND): a randomised, open-label phase 3 trial. Lancet Oncol 14: 490-499, 2013.

44. Hu CJ, Wang B, Tang B, Chen BJ, Xiao YF, Qin Y, Yong X, Luo G, Zhang JW, Zhang D, et al: The FOXM1-induced resistance to oxaliplatin is partially mediated by its novel target gene Mcl-1 in gastric cancer cells. Biochim Biophys Acta 1849: 290-299, 2015.

45. Park YH, Kim BS, Ryoo BY and Yang SH: A phase II study of capecitabine plus 3-weekly oxaliplatin as first-line therapy for patients with advanced gastric cancer. Br J Cancer 94: 959-963, 2006.

46. Harada S, Yanagisawa M, Kaneko S, Yorozu K, Yamamoto K, Moriya Y and Harada N: Superior antitumor activity of trastuzumab combined with capecitabine plus oxaliplatin in a human epidermal growth factor receptor 2-positive human gastric cancer xenograft model. Mol Clin Oncol 3: 987-994, 2015.

47. Soularue É, Cohen R, Tournigand C, Zaanan A, Louvet C, Bachet JB, Hentic O, Samalin E, Chibaudel B, de Gramont A and André T; for GERCOR: Efficacy and safety of trastuzumab in combination with oxaliplatin and fluorouracil-based chemotherapy for patients with HER2-positive metastatic gastric and gastro-oesophageal junction adenocarcinoma patients: a retrospective study. Bull Cancer 102: 324-331, 2015. 